\title{
Article \\ Common Perceptions about the Use of Fillers in Silicone Rubber Insulation Housing Composites
}

\author{
Refat Atef Ghunem *(D, Yazid Hadjadj and Harold Parks \\ Metrology Research Center, National Research Council Canada, 1200 Montreal Rd., \\ Ottawa, ON K1A0R6, Canada; Yazid.Hadjadj@nrc-cnrc.gc.ca (Y.H.); Harold.Parks@nrc-cnrc.gc.ca (H.P.) \\ * Correspondence: Refat.Ghunem@nrc-cnrc.gc.ca
}

check for updates

Citation: Ghunem, R.A.; Hadjadj, Y.; Parks, H. Common Perceptions about the Use of Fillers in Silicone Rubber Insulation Housing Composites. Energies 2021, 14, 3655. https:// doi.org/10.3390/en14123655

Academic Editors: Pawel Rozga and Zhijin Zhang

Received: 28 April 2021

Accepted: 16 June 2021

Published: 19 June 2021

Publisher's Note: MDPI stays neutral with regard to jurisdictional claims in published maps and institutional affiliations.

Copyright: (c) 2021 by the authors. Licensee MDPI, Basel, Switzerland. This article is an open access article distributed under the terms and conditions of the Creative Commons Attribution (CC BY) license (https:// creativecommons.org/licenses/by/ $4.0 /)$.

\begin{abstract}
This paper discusses the lessons learned about fillers that are added to silicone rubber insulation housing composites, in order to improve the erosion resistance against the dry-band arcing are presented and common perceptions. Common practices that employ alumina tri-hydrate are reviewed, including the additional influential effect of the water of hydration in the suppression of the dry-band arcing. The effect of the water of hydration is shown to be dependent on various factors, such as the hydrated filler level and the type of the hydrated filler. More recent paradigms in which hydrated fillers have not been employed are also reviewed. Volume and shield actions of fillers are essential aspects that need to be understood in the design of silicone rubber insulation housing composites for new applications such as HVDC. In addition, the thermal degradation mechanisms of silicone rubber and the corresponding suppression effects of the added fillers are summarized.
\end{abstract}

Keywords: silicone rubber; outdoor insulators; transmission and distribution; pollution performance; tracking; erosion resistance

\section{Introduction}

Silicone rubber insulators are replacing the conventional ceramic insulators due to their proven performance when exposed to environmental pollutants. Silicone rubber has shown a unique ability to wash off contaminants and retain the hydrophobicity of the insulator, due to a low-molecular-weight (LMW) siloxane diffusing from the insulator housing bulk to the surface. Therefore, silicone rubber insulators have become backbone components of the overhead power delivery infrastructure. However, after aging in service, leakage currents may still develop under the effect of voltage, moisture and contamination, causing the depletion of the LMW siloxane, and thus, deteriorating the hydrophobicity recovery of the insulator [1]. Eventually dry-band arcing arises, causing heat ablation, which exposes the insulator fiberglass core to weathering and the consequent failure of the entire insulator due to tracking of the core (Figure 1) [2].

Fillers are added to silicone rubber outdoor insulation housing composites in order to enhance the erosion resistance, but the primary purpose of using fillers is cost reduction achieved by replacing the expensive silicone with the filler particles. Cost reductions have been a great incentive for loading silicone rubber outdoor insulation composites with as much filler as possible. However, this approach also reduces the amount of the LMW siloxane responsible for recovering the hydrophobicity of silicone rubber [1]. In addition, the cost of the fillers may vary depending on the type of filler. Therefore, achieving an optimized composite is a complex problem. 


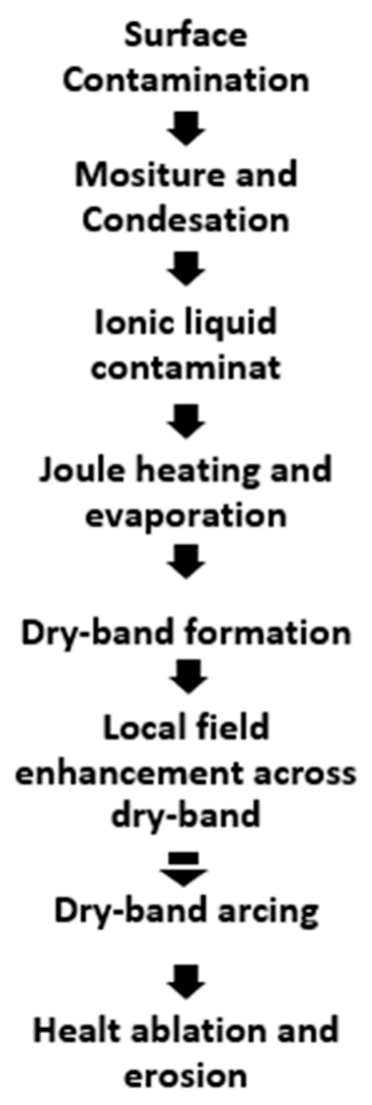

Figure 1. Erosion mechanism on the surface of silicone rubber outdoor insulation housing.

The application of fillers in silicone rubber outdoor insulator composites is usually based on experience, which has been so far successful as demonstrated by decades of proven performance in AC insulation applications. This successful experience has been used to justify the application of existing insulator composite designs for newer applications such as high voltage direct current (HVDC) outdoor insulation. However, recent finding raise concerns about this approach for new and different application under HVDC. Experimental findings and field observations have reported a more severe erosion under DC voltages as compared to AC voltages [3,4]. The HVDC power system has become an essential building block of the power transmission and distribution infrastructure in the green electricity grid. Therefore, it is essential to address concerns related to DC insulator aging by investigating the suitable use of fillers at the material development stage. In general, little attention has been given to the housing material design aspects as compared to the geometry aspect of silicone rubber insulators. While a geometric design has been mainly correlated with the insulator flashover performance, the use of fillers mainly controls the erosion performance of the insulator [1]. This paper summarizes the evolution of the design paradigms in the development of erosion-resistant silicone rubber insulation housing composites by shedding light on the common perceptions about the application of inorganic fillers. In particular, critical discussion of re-applying perceptions, developed under AC conditions for new applications, such as HVDC outdoor insulation is presented and lessons learned are summarized.

\section{Tracking and Erosion of Silicone Rubber}

The terms tracking and erosion refer to two different surface failure mechanisms of insulators and therefore should not be interchangeably used. Tracking usually refers to the formation of a carbonaceous path that could be fully or partially conductive on the surface of the insulation housing. Erosion of silicone rubber insulators may be defined as heat ablation of the insulation housing as a result of surface discharges in general or 
dry-band arcing in particular impinging the surface. It must be emphasized that silicone rubber has shown a tracking-resistant behavior during material screening tests [3,5]. Unlike many other outdoor insulating materials, silicone rubber possesses a backbone that does not intrinsically promote a significant amount of carbon residue when exposed to surface discharges in wet conditions [5]. Although it is reported to be susceptible to tracking in dry-conditions, silicone rubber without hydrated fillers have shown acceptable tracking performance in wet conditions [5]. In addition, silicone rubber does not leave a significant amount of carbonaceous residue during dry-band arcing [6]. Both tracking and erosion have been shown to be stimulated as a result of the dry-band arcing heat, but they are a result of two different thermal degradation mechanisms of silicone rubber. Erosion has been shown initiated with the depolyemrization of the silicone rubber, through secession of Si-O (siloxane) bond, yielding cyclic oligomers with small molecular weights and low flashpoints in the gas phase [7]. Tracking is promoted in silicone rubber as a result of the radical-based crosslinking [8], initiated with the $\mathrm{CH}_{3}$-Si bond scission in silicone rubber [9]. Therefore, tracking as an aging mechanism must not be conflated with erosion for silicone rubber insulators. Yet, this understanding has not been emphasized in the literature despite decades of experience.

Understanding the aging mechanisms leading to erosion is an essential task to support the proper application of inorganic fillers in silicone rubber insulators. The two main thermal degradation mechanisms of silicone rubber can be identified using thermogravimetric analysis (TGA) and the derivative thermogravimetric analysis (DTGA). Figure 2 is an illustrative example shown for a TGA-DTGA conducted on RTV silicone rubber composite in Nitrogen atmosphere (test details in the caption). Two main weight loss stages are observed in Figure 2 for silicone rubber, indicating two distinct mechanisms governing erosion. The first weight loss stage begins at $350{ }^{\circ} \mathrm{C}$ and is due to depolymerization, i.e., due to scission, of the siloxane bonds. The second weight loss stage, beginning at $600{ }^{\circ} \mathrm{C}$, is associated to another distinct peak in the DTGA, indicating occurrence of another degradation mechanism, i.e., radical-based crosslinking [8,9]. Depolymerization is mainly responsible for weight loss and thus erosion during the dry-band arcing [7]. Figure 3 illustrates a simplified diagram for the tracking and erosion mechanisms of silicone rubber.

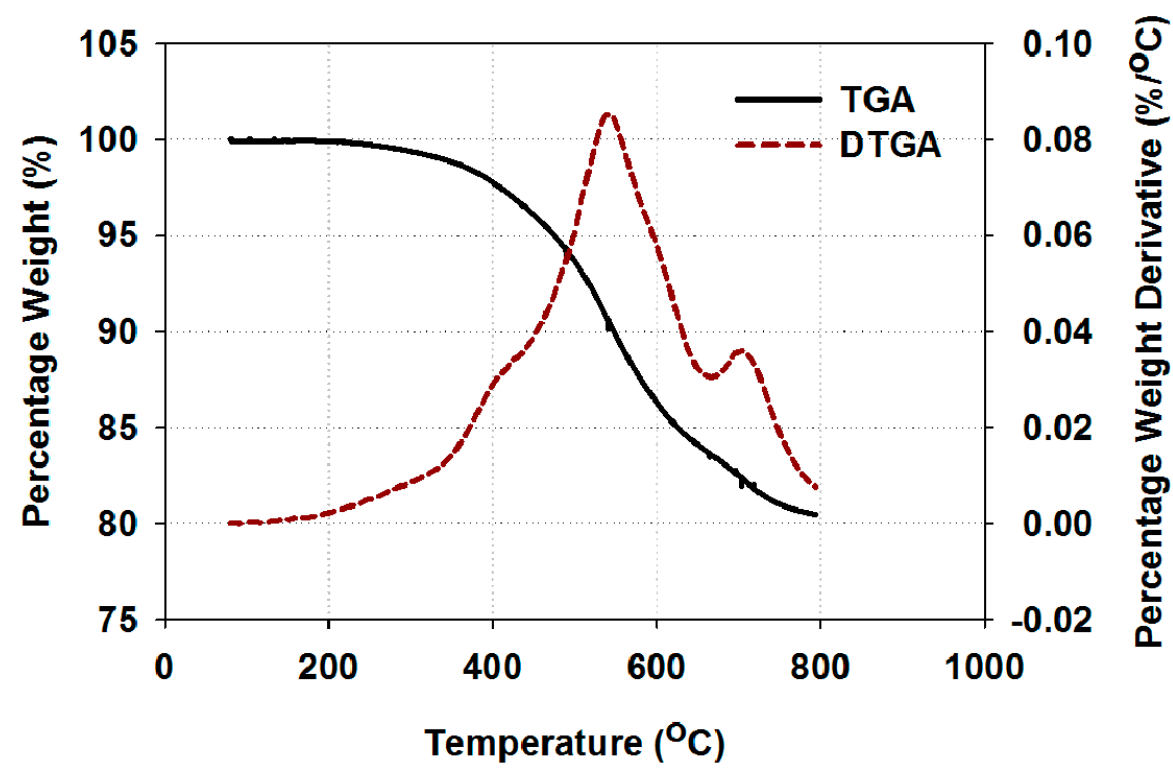

Figure 2. TGA of RTV silicone rubber filled with natural silica to $50 \mathrm{wt}$. $\%$, performed in Nitrogen atmosphere under a temperature ramping protocol between 80 and $800^{\circ} \mathrm{C}$ at a rate of $10^{\circ} \mathrm{C} / \mathrm{min}$. 


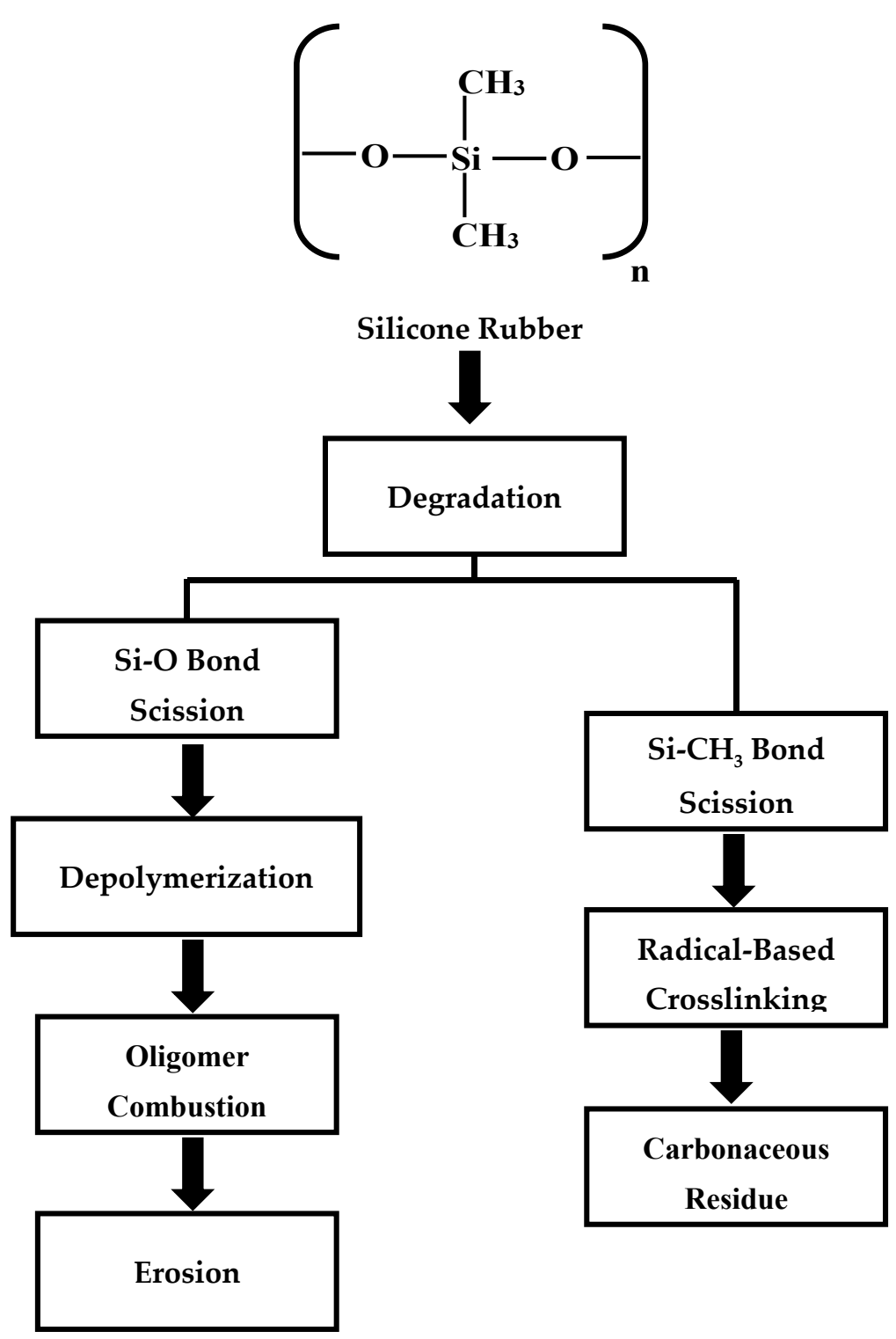

Figure 3. Thermal degradation mechanisms promoting carbonaceous residue and erosion of silicone rubber outdoor insulation.

The relative degree of crosslinking can be estimated for the silicone rubber composite, given the filler level added should be known, using the final residue level in the TGA. This estimation is useful in understanding the filler protective mechanisms against the degradation mechanisms of silicone rubber [8]. For example, the total amount of residue obtained after the TGA for the silicone rubber composite shown in Figure 2 is about $80 \mathrm{wt}$ \%. Given the silicone rubber base is unfilled [10], this residue constitutes of the filler amount, i.e., $50 \mathrm{wt}$ \% and the remaining $30 \mathrm{wt}$ \% is additional residue formed due to radical-based crosslinking. Note that this estimation method assumes a negligible amount of other constituents, such as crosslinking agents or pigments. It should be noted that the water of hydration amount should be taken into consideration in the estimation if the filler is hydrated. Figure 4 shows the TGA-DTGA conducted in Nitrogen atmosphere for RTV silicone rubber filled with ATH to $50 \mathrm{wt}$.\% (test details in caption). Similar to the silica-filled RTV silicone rubber, the ATH-filled RTV silicone rubber also showed a depolymerization stage beginning at $350{ }^{\circ} \mathrm{C}$ and a crosslinking beginning at $550{ }^{\circ} \mathrm{C}$. An additional weight loss stage is seen with ATH before depolymerization, starting at $200{ }^{\circ} \mathrm{C}$, which has been attributed to the dehydration of ATH [11]. Typically, ATH in silicone rubber releases about 
35 wt.\% of the total filler amount during TGA [11], so the amount of the water of hydration released from the composite is estimated to be about $17.5 \mathrm{wt} \%$ and the remaining amount of alumina particles are $32.5 \mathrm{wt}$.\%. The final TGA residue level is about $66 \mathrm{wt} . \%$. With alumina particles estimated to be about $32.5 \mathrm{wt} . \%$, the amount of the crosslinked silicone rubber is estimated to be $33.5 \mathrm{wt}$.\%.

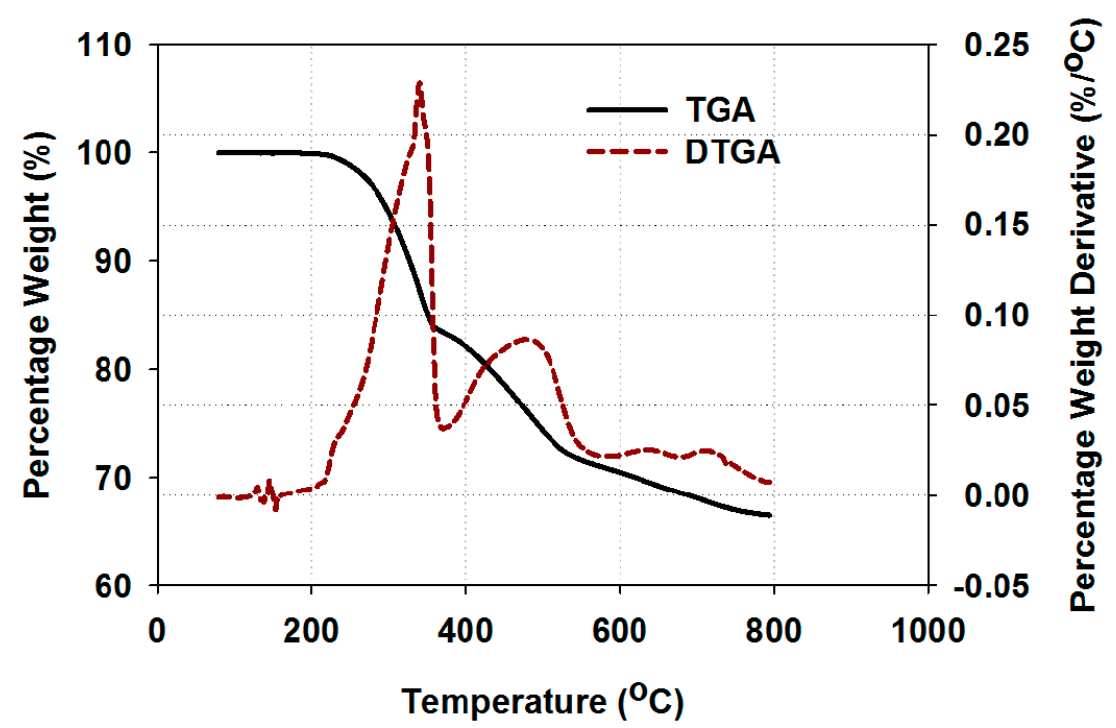

Figure 4. TGA-DTGA of RTV silicone rubber filled with ATH to $50 \mathrm{wt}$.\%, performed in Nitrogen atmosphere under a temperature ramping protocol between 80 and $800{ }^{\circ} \mathrm{C}$ at a rate $10{ }^{\circ} \mathrm{C} / \mathrm{min}$.

Often, fumed silica is added to the silicone base, in order to enhance the mechanical properties of the silicone rubber insulation housing. This fumed silica has a different purpose and is added in smaller amount than either ground silica or ATH, which are added to reduce the cost and enhance the erosion resistance. If the silicone base contains fumed silica, the corresponding base residue would include fumed silica particles and some crosslinked residue is formed as a result of polymer-filler interactions between the base and the fumed silica. As such, this base residue needs to be taken into account, by running first TGA for silicone base without the ground silica or ATH filler and the amount of the base residue should be noted. Accordingly, the percentage of the crosslinked residue due to ground silica or ATH in the composite can be estimated by subtracting from the percentage of the final TGA residue of the composite: (1) Percentage of the ground silica (or alumina level if ATH is added), (2) percentage of the water of hydration released if ATH is added and the percentage amount of the base residue obtained from the first TGA multiplied by the fraction of the actual base silicone rubber in composite. Details about this estimation method can be found in [12].

\section{Fillers in Silicone Rubber Outdoor Insulation Composites}

Mineral fillers were initially employed in polymeric insulating materials in order to reduce the organic content that promotes tracking. This reduction in organic content inhibits the tendency of the insulator to form a carbonaceous residue during thermal decomposition when the insulator was impinged by dry-band arcs [5]. However, different outcomes were reported under wet as compared to dry conditions [5]. In particular, mineral fillers were not shown effective in inhibiting tracking in wet conditions. It should be noted that wet laboratory conditions better simulate the outdoor insulation environment than dry conditions. The use of hydrated fillers such as alumina tri-hydrate (ATH) was later proposed. It was shown that the released water of hydration during surface discharges stimulates an internal oxidation between the organic backbone of the insulation and the hydroxyl groups released from ATH, yielding carbon oxides and hydrocarbons in the gas phase, instead of leaving a carbonaceous track on the surface $[13,14]$. In addition, the 
released water of hydration as vapor was reported to physically promote sputtering of any traces of carbonaceous tracks formed on the surface, thereby preventing the formation of a continuous conductive path that may lead to insulator failure [15].

Some concerns were initially raised about the erosion taking place in addition to tracking of insulating materials, but the suppression of tracking was clearly the main concern at the early times. The focus on tracking was probably because most of the outdoor insulation materials had organic backbones. Even material screening methods were primarily developed as tests for tracking with less emphasis on erosion [16]. Later on, the need to evaluate erosion was highlighted [17].

Today, ATH is the main filler added to silicone rubber and makes up about $60 \mathrm{wt} . \%$ of the formulation, in order to achieve acceptable performance in the outdoors. This performance is verified at the material development stage in the inclined plane tracking and erosion test (IPT) at $4.5 \mathrm{kV}$ as per ASTM D2303 or IEC 60587, i.e., the critical $\mathrm{AC}_{\mathrm{rms}}$ voltage level $[7,18]$. It is unclear why, specifically, $60 \mathrm{wt} . \%$ of ATH was decided upon, but this approach seems to be come from the use of ATH as a flame retardant in silicone rubber formulations for different applications [19].

The use in ATH at $60 \mathrm{wt} . \%$ has been successfully used for decades in various HVAC outdoor insulation applications. Therefore, there has been little motivation for the development of improved or cost-reduced designs of silicone rubber composites for HVAC insulation and only a few alternatives to ATH in silicone rubber have been proposed. However, hotspots causing erosion has been highlighted as an issue that needs to be suppressed. As such, the increase in the thermal conductivity of the overall composite has been used as an indicator for the efficacy of the fillers to suppress erosion. Silica been shown to offer a similar increase in thermal conductivity of silicone rubber to ATH, but at fraction of the overall cost of the composite [20]. The use of ATH was shown to promote a layered-residue on the surface during surface discharges, which led to the failure in the IPT at the critical voltage [21]. The layered residue consists of mullite formed when hotspots temperatures exceeding $1200{ }^{\circ} \mathrm{C}$ on the surface [6,21]. The UL94 burning test was also employed to test silicone rubber composites filled with alternative fillers to ATH. Both silica and melamine cyanurate were shown to enhance the ability of silicone rubber to extinguish arcs on the surface. In addition, three main design aspects were highlighted as areas that need to be taken into account: Filler level, good dispersion and bonding between host matrix and filler particles [21].

These existing paradigms need to be critically examined when designing formulas for applications where there is limited experience from the field such as silicone rubber insulators for HVDC lines. The nature of the dry-band arcing under DC has been shown in different tests to be more continuous and intense than under $\mathrm{AC}$ conditions, leading to deep erosion [22]. Therefore, the need to develop specialized silicone rubber composites for HVDC has become an important issue. The use of fillers as flame-retardants has been discussed for HVDC silicone rubber insulation [23], highlighting two main aspects; (1) the suppression of the heat source, i.e., surface discharge causing erosion and (2) the suppression of the thermal degradation leading to erosion of silicone rubber. The first aspect has been investigated extensively, and it is known that ATH impedes the induction hotspots in both the solid and the gas phase of silicone rubber [23,24]. However, the influential of ATH on the second more important aspect, the suppression of thermal degradation, has yet to be investigated.

\section{Design Aspects}

\subsection{Volume Effect of Fillers}

The term "volume effect" refers to the filler volume replacing the amount of the polymeric fuel prone to degradation during the dry-band arcing. This paradigm may be understood as analogous to the application of fillers as flame retardants in polymers. It has been understood that adding as much filler as possible would reduce the amount of the carbonaceous content that can lead to tracking of the polymer. However, tracking of 
polymeric insulating materials has not been shown to necessarily depend on the amount of the organic content [25]. In addition, applying fillers to inhibit tracking may not necessarily suppress erosion failure. For example, silica-filled silicone rubber impinged with the dryband arcing did not track in the IPT, but failed due to erosion paths [10]. In particular, the combustion of silcione rubber oligomers produced from depolymerization may have promoted electrical breakdown in the gas phase [6]. The electrical breakdown in the gas phase has been detected using wavelet-based analysis of the leakage current measured in-situ during the IPT [22].

On the other hand, this failure pattern has not been observed for the RTV silicone rubber containing same amount of ATH [10]. It has been shown that a critical ATH level of $45 \mathrm{wt} . \%$ is needed in silicone rubber in order to promote an internal oxidation that could help suppress failure in the IPT [7]. At the same loading glevel, both silica and ATH have been shown to increase the thermal conductivty of silicone rubber to a similar extent. On the ther hand, an additional influence could be expected for the water of hydration in the gas phase. The use of ATH may inturupt the combustion cycle of silicone rubber by dilluting the gas phase of silicone rubber through internal oxidation $[7,23]$. The internal oxdiation effect has been detected in the DTA with a clear suppression in the exothermic hump obtained in TGA performed in air after the depolymerization of silicone rubber [7].

\subsection{Residue Shield}

Ground silica is not a hydrated filler and therefore cannot dilute the gas phase of silicone rubber during the dry-band arcing. Therefore, it cannot be accurately concluded that ATH would be have been more effective, and thus, better in preventing failure of RTV silicone rubber in the IPT than silica. However, when a larger particle size of silica was used at the same level of $50 \mathrm{wt} . \%$, the RTV silicone rubber composite withstood the $4.5 \mathrm{kV} \mathrm{AC}$ voltage in the IPT [8]. Increasing the size of silica has been shown to lead to a more coherent residue shield against the dry-band arcing [8]. It has also been shown that the coherence of the residue shield against the dry-band arcing is an essential factor for the suppression of the DC dry-band arcing [8]. Also, the water arising from the hydration released from ATH filler in silicone rubber may leave porous residue during thermal degradation [11,19]. It has been suggested that using an ATH filler with a small particle size about $0.3 \mu \mathrm{m}$ promotes hydrolysis that could disadvantageously cause erosion during DC dry-band arcing [11].

Another important example is the comparison of the water of hydration effect of ATH and magnesium hydroxide (MH). It has been widely understood that the enthalpy of dehydration of hydrated fillers such as ATH and Magnesium hydroxide fillers would be an important property, governing the flame retardancy of the hydrated filler. The $\mathrm{MH}$ has shown comparable enthalpy of dehydration to ATH [11]. The $\mathrm{MH}$ in silicone rubber dehydrates during the depolymerization of silicone rubber. Whereas, ATH dehydrates before the depolymerization [11]. Figure 5 illustrate an example of TGA-DTGA conducted for RTV silicone rubber filled with $\mathrm{MH}$ to $50 \mathrm{wt}$ \% in Nitrogen atmosphere (test details in the caption), showing only one weight loss stage before radical-based crosslinking (at $<600^{\circ} \mathrm{C}$ ), which indicates both dehydration of $\mathrm{MH}$ and depolymerization of silicone rubber take place during at the same weight loss stage. Based on such an understanding, similar or even better erosion suppression would be expected for $\mathrm{MH}$, as compared to ATH in silicone rubber. This outcome has been reported under AC voltages [10]. However, silicone rubber filled with ATH has led to a significantly better erosion resistance than silicone rubber filled with same level of $\mathrm{MH}$ under DC voltages [11]. This finding could be a result of dehydration of ATH taking place prior to depolymerization of silicone rubber at $400{ }^{\circ} \mathrm{C}$ has allowed a more coherent protective shield of alumina to form against the dry-band arcing as compared to Magnesium hydroxide that leaves a more porous shield against the DC dry-band arcing after dehydration [11] 


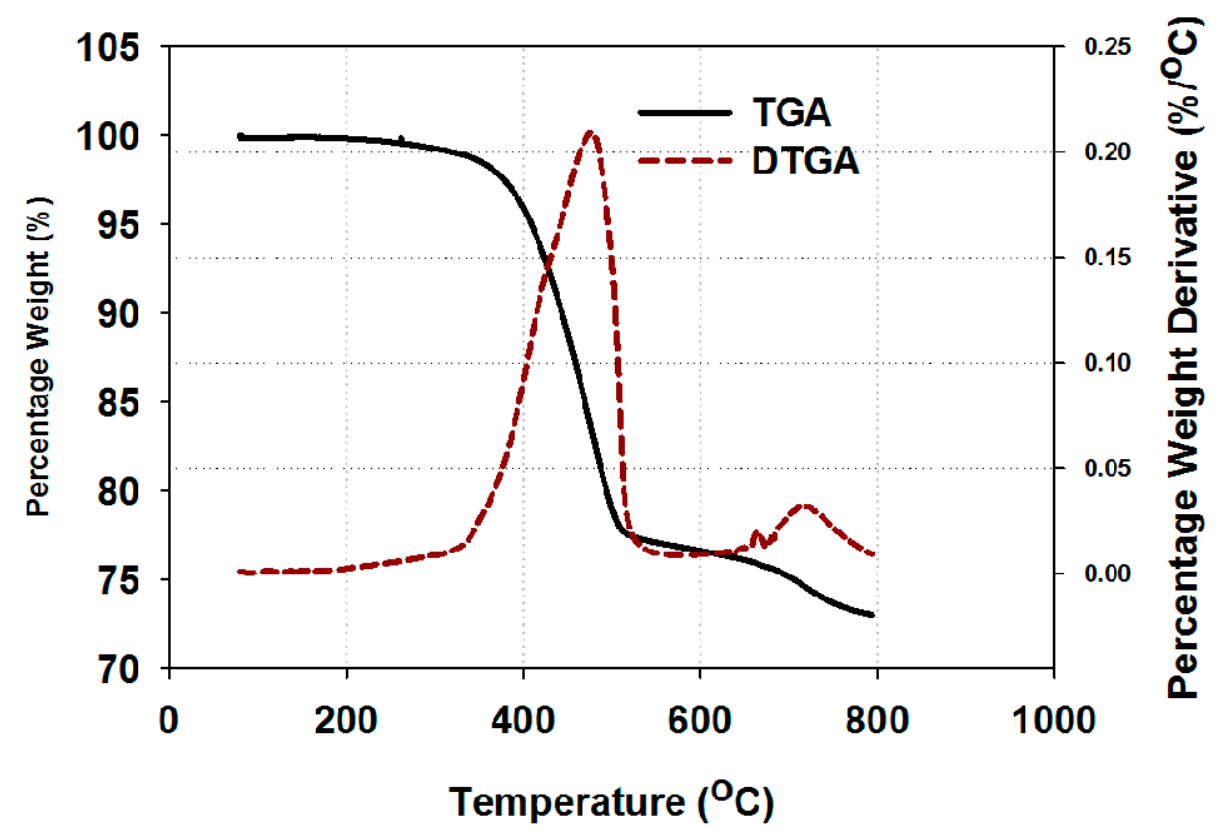

Figure 5. TGA-DTGA of RTV silicone rubber filled with $\mathrm{MH}$ to $50 \mathrm{wt}$.\%, performed in Nitrogen atmosphere under a temperature ramping protocol between 80 and $800{ }^{\circ} \mathrm{C}$ at a rate of $10^{\circ} \mathrm{C} / \mathrm{min}$.

Therefore, it must be emphasized that different filler actions may be differently utilized in order to achieve acceptable erosion performance. In addition, the claim that a filler type is superior to another type must not be made while only looking at a single filler action or design aspect. It is important to understand the different types of filler actions that suppress the erosion mechanisms of silicone rubber.

\section{Screening Methods at the Material Development Stage}

The IPT is the most widely accepted test for the evaluation of erosion resistance of silicone rubber composites for outdoor AC insulation applications when conducted as per ASTM D2303 or IEC 60587. The test has been modified to suit testing under DC voltages. However, the proper implementation of IPT test methods is still an essential task in the evlauation of silicone rubber composites. Otherwise, the outcomes reported may be misleading [15]. In particular, the constant voltage method is a more suitable method when testing for tracking-resistant materials such as silicone rubber. Whereas, the stepwise voltage method would be a more suitable method to test for tracking, progressive erosion or to determine the suitable test voltage for the costant voltage method $[3,15]$.

Moreover, it is useful to develop other test methods for the erosion resistance that can provide more insight into the filler actions, particualy at the material development stage. Such considerations are very important for testing under DC voltages, given the relatively limited experience with testing under DC voltages. The application the IPT has been extended by applying a leakage current-based technique to compare the initation of eroding hotspots due to the DC dry-band arcing [22]. With the effect of the filler on the depolymerization and radical-based crosslinking of silicone rubber, the use of the TGA and DTA, thermal conducivity measurements and the scanning electron microspcopy (SEM) has been reported $[8,11,12]$.

\section{Conclusions}

Perceptions and design paradigms about the development of erosion resistant silicone rubber insulators for outdoor insulation using inorganic fillers have been discussed and lessons learned are summarized. The concept of tracking resistance must be recognized as distinct from erosion resistance for silicone rubber. Two aspects must be taken into account when designing erosion resistant silicone rubber insulation housing composites, namely 
the volume effect of the fillers and the shield effect. In addition the methods of testing the design must be well considered. Supplementary material characterization tests and measurements such as TGA, SEM, leakage current measurements and thermal conductivity measurements are useful in understanding the protective filler actions in enhancing the erosion resistance of silicone rubber.

Author Contributions: Conceptualization, R.A.G., Y.H. and H.P; writing—original draft preparation, R.A.G.; writing-review and editing, R.A.G., H.P., and Y.H.; visualization, R.A.G.; supervision, H.P.; project administration, R.A.G.; All authors have read and agreed to the published version of the manuscript.

Funding: This research received no external funding.

Conflicts of Interest: The authors declare no conflict of interest.

\section{References}

1. Gorur, R.; Burnham, J.T.; Cherney, E.A. Outdoor Insulators; Ravi S. Gorur Inc.: Phoenix, AZ, USA, 1999.

2. Ghunem, R. A Study of the Erosion Mechanisms of Silicone Rubber Housing Composites. Ph.D. Thesis, ECE University Waterloo, Waterloo, ON, Canada, 2014.

3. Ghunem, R.; Jayaram, S.H.; Cherney, E.A. Erosion of silicone rubber composites in the AC and DC inclined plane tests. IEEE Trans. Dielectr. Electr. Insul. 2013, 20, 229-236. [CrossRef]

4. Liang, X.; Li, S.; Gao, Y.; Su, Z.; Zhou, J. Improving the outdoor insulation performance of Chinese EHV and UHV AC and DC overhead transmission lines. IEEE Electr. Insul. Mag. 2020, 36, 7-25. [CrossRef]

5. Sommerman, G.M.L. Electrical Tracking Resistance of Polymers. Trans. AIEE Part III Power Appar. Syst. 1960, 79, 969-974. [CrossRef]

6. Kumagai, S.; Wang, X.; Yoshimura, N. Solid residue formation of RTV silicone rubber due to dry-band arcing and thermal decomposition. IEEE Trans. Dielectr. Electr. Insul. 1998, 5, 281-289. [CrossRef]

7. Kumagai, S.; Yoshimura, N. Tracking and erosion of HTV silicone rubber and suppression mechanism of ATH. IEEE Trans. Dielectr. Electr. Insul. 2001, 8, 203-211. [CrossRef]

8. Koné, D.; Ghunem, R.A.; Cissé, L.; Hadjadj, Y.; El-Hag, A.H. Effect of residue formed during the AC and DC dry-band arcing on silicone rubber filled with natural silica. IEEE Trans. Dielectr. Electr. Insul. 2019, 26, 1620-1626. [CrossRef]

9. Delebecq, E.; Hamdani-Devarennes, S.; Raeke, J.; Lopez Cuesta, J.M.; Ganachaud, F. High Residue Contents Indebted by Platinum and Silica Synergistic Action During the Pyrolysis of Silicone Formulations. ACS Appl. Mater. Interfaces 2011, 3, 869-880. [CrossRef] [PubMed]

10. Ghunem, R.A.; El-Hag, A.H.; Hadjadj, Y.; Cherney, E.A. A study on the effect of inorganic fillers on the dry-band arcing erosion of silicone rubber composites. In 2017 IEEE Conference on Electrical Insulation and Dielectric Phenomenon (CEIDP); IEEE: Piscataway, NJ, USA, 2017; pp. 664-667.

11. Ghunem, R.; Koné, D.; Cissé, L.; Hadjadj, Y.; Parks, H.; Ambroise, D. Effect of hydrated fillers in silicone rubber composites during AC and DC dry-band arcing. IEEE Trans. Dielectr. Electr. Insul. 2020, 27, 249-256. [CrossRef]

12. El-Hag, A.; Simon, L.C.; Jayaram, S.H.; Cherney, E.A. Erosion resistance of nano-filled silicone rubber. IEEE Trans. Dielectr. Electr. Insul. 2006, 13, 122-128. [CrossRef]

13. Norman, R.; Kessel, A. Mechanism for nontracking organic insulations. Electr. Eng. 1958, 77, 699. [CrossRef]

14. Norman, R.; Kessel, A. Internal Oxidation Mechanism for Nontracking Organic Insulations. Trans. AIEE Part III Power Appar. Syst. 1958, 77, 632-636. [CrossRef]

15. Ghunem, R. Using the inclined-plane test to evaluate the resistance of outdoor polymer insulating materials to electrical tracking and erosion. IEEE Electr. Insul. Mag. 2015, 31, 16-22. [CrossRef]

16. Mathes, K.; Mcgowan, E. Surface electrical failure in the presence of contaminants: The inclined-plane liquid-contaminant test. Trans. AIEE Part I Commun. Electron. 1961, 80, 281-289. [CrossRef]

17. Mathes, K.; Mcgowan, E. Erosion-Inclined plane, liquid contaminant tracking test. In Proceedings of the Annual Report 1961 Conference on Electrical Insulation, Pocono Manor, PA, USA, 23-25 October 1961; pp. 121-124.

18. Krivda, A.; Krivda, A.; Schmidt, L.E.; Kornmann, X.; Ghorbani, H.; Ghorbandaeipour, A.; Eriksson, M.; Hillborg, H. Inclined-plane tracking and erosion test according to the IEC 60587 standard. IEEE Electr. Insul. Mag. 2009, 25, 14-22. [CrossRef]

19. Hamdani, S.; Longuet, C.; Perrin, D.; Lopez-Cuesta, J.M.; Ganachaud, F. Flame retardancy of silicone-based materials. Polym. Degrad. Stab. 2009, 94, 465-495. [CrossRef]

20. Meyer, L.; Cherney, E.A.; Jayaram, S.H. The role of inorganic fillers in silicone rubber for outdoor insulation alumina tri-hydrate or silica. IEEE Electr. Insul. Mag. 2004, 20, 13-21. [CrossRef]

21. Schmidt, L.; Kornmann, X.; Krivda, A.; Hillborg, H. Tracking and erosion resistance of high temperature vulcanizing ATH-free silicone rubber. IEEE Trans. Dielectr. Electr. Insul. 2010, 17, 533-540. [CrossRef]

22. Ghunem, R.; Jayaram, S.H.; Cherney, E.A. Investigation into the eroding dry-band arcing of filled silicone rubber under DC using wavelet-based multiresolution analysis. IEEE Trans. Dielectr. Electr. Insul. 2014, 21, 713-720. [CrossRef] 
23. Ghunem, R.; Jayaram, S.H.; Cherney, E.A. The DC inclined-plane tracking and erosion test and the role of inorganic fillers in silicone rubber for DC insulation. IEEE Electr. Insul. Mag. 2015, 31, 12-21. [CrossRef]

24. Ghunem, R.; Jayaram, S.H.; Cherney, E.A. Suppression of silicone rubber erosion by alumina trihydrate and silica fillers from dry-band arcing under DC. IEEE Trans. Dielectr. Electr. Insul. 2015, 22, 14-20. [CrossRef]

25. Billings, M.; Smith, A.; Wilkins, R. Tracking in Polymeric Insulation. IEEE Trans. Electr. Insul. 1967, EI-2, 131-137. [CrossRef] 